\title{
k-cosymplectic manifolds and Lagrangian field theories
}

\author{
Manuel de León, ${ }^{\text {a) }}$ Eugenio Merino, ${ }^{\text {b) }}$ and Modesto Salgado ${ }^{c)}$ \\ Consejo Superior de Investigaciones Cientificas, Instituto de Matematicas y Fisica \\ Fundamental, Serrano 123, 28006 Madrid, Spain
}

(Received 14 September 2000; accepted for publication 29 January 2001)

A geometrical description of classical field theories of first order is given. The underlying $k$-cosymplectic structure permits to derive the corresponding field equations. () 2001 American Institute of Physics. [DOI: 10.1063/1.1360997]

\section{INTRODUCTION}

As is well-known, the natural arena to study mechanics is symplectic geometry. One interesting problem is to extend this geometric framework for the case of classical field theories. Several different approaches were developed in the past forty years, and according to them, the field equations are presented in different geometric formats: jet fields, Ehresmann connections, or multivector fields (we refer the reader to Refs. 1-5 and the references therein; see also Refs. 6-21 for recent developments).

In a recent paper $^{22}$ we have constructed a geometric description of classical field theories in terms of $k$-cosymplectic manifolds. A $k$-cosymplectic manifold is a natural generalization of a cosymplectic manifold, which is the geometrical setting for nonautonomous mechanical systems (see Refs. 23-25).

In the present paper, which can be viewed as a natural extension of Ref. 22, we consider the Lagrangian description of first order classical field theories. The field equations are then derived by using the canonical almost stable $k$-tangent structure on the stable $k$-tangent bundle $\mathbf{R}^{k} \times T_{k}^{1} Q$ of a manifold $Q$. From the Lagrangian function $L$, we construct in an intrinsical way the Poincaré-Cartan forms $\left(\omega_{\mathrm{L}}\right)_{A}, 1 \leqslant A \leqslant k$, which determine a $k$-cosymplectic structure if $L$ is regular. The $k$-cosymplectic structure provides us a $k$-vector field whose integral sections are the solutions of the field equations. The integrability is not assured, but it can be checked in terms of an arbitrary solution.

If the Lagrangian is not regular, then we develop a constraint algorithm which, if the field equations are compatible, yields a final constraint submanifold where a solution exists. This algorithm is very similar to that constructed in Ref. 25.

This Lagrangian description is of course completely equivalent to the Hamiltonian one developed in our precedent paper by means of a convenient Legendre transformation.

\section{II. $\boldsymbol{k}$-VECTOR FIELDS}

Let $M$ be a differentiable manifold of dimension $m$ and $\mathbf{R}^{\mathbf{k}}$ the $k$-dimensional Euclidean space with coordinates $t=\left(t^{A}\right)=\left(t^{1}, \ldots, t^{k}\right)$. Let $J_{0}^{1}\left(\mathbf{R}^{\mathbf{k}}, M\right)$ be the $(k+1) m$-dimensional manifold of one jet of mappings from $\mathbf{R}^{\mathbf{k}}$ to $M$ at the origin of $\mathbf{R}^{\mathbf{k}}$ with elements denoted by $j_{0}^{1} \sigma . J_{0}^{1}\left(\mathbf{R}^{\mathbf{k}}, M\right)$ is called the tangent bundle of $k^{1}$-velocities in Ref. 26.

Let $\left\{\mathbf{r}_{1}, \ldots, \mathbf{r}_{\mathbf{k}}\right\}$ be the canonical basis of $\mathbf{R}^{\mathbf{k}}$. The manifold $J_{0}^{1}\left(\mathbf{R}^{\mathbf{k}}, M\right)$ can be canonically identified with the Whitney sum $T_{k}^{1} M=T M \oplus \cdots \oplus T M$ of $k$ copies of $T M$ via the diffeomorphism,

$$
J_{0}^{1}\left(\mathbf{R}^{\mathbf{k}}, M\right) \rightarrow T_{k}^{1} M=T M \oplus \cdots \oplus T M
$$

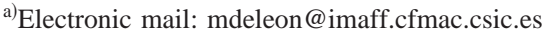

${ }^{b)}$ Electronic mail: uxiomer@eps.cdf.udc.es

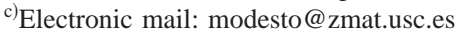




$$
j_{0}^{1} \sigma \rightarrow\left(v_{1}, \ldots, v_{k}\right)
$$

defined by

$$
v_{A}=\left.\frac{d \sigma_{A}}{d s}\right|_{s=0}, \quad s \in \mathbf{R}, \quad 1 \leqslant A \leqslant k,
$$

where $\sigma_{A}(s)=\sigma\left(s \mathbf{r}_{\mathbf{A}}\right)$.

Let $\tau_{M}: T_{k}^{1} M \rightarrow M$ be the canonical projection. If $\left(x^{i}\right)$ are local coordinates on $U \subseteq M$ then the induced local coordinates $\left(x^{i}, x_{A}^{i}\right), 1 \leqslant i \leqslant m, 1 \leqslant A \leqslant k$, on $\tau_{M}^{-1}(U) \equiv T_{k}^{1} U$ are given by

$$
x^{i}\left(j_{0}^{1} \sigma\right)=x^{i}(\sigma(0)), \quad x_{A}^{i}\left(j_{0}^{1} \sigma\right)=\frac{d}{d s}\left(x^{i} \circ \sigma^{A}\right)_{\mid s=0}=v_{A}\left(x^{i}\right) .
$$

Definition II.1: A section $\mathbf{X}: M \rightarrow T_{k}^{1} M$ of the projection $\tau_{M}: T_{k}^{1} M \rightarrow M$ will be called a $k$-vector field on $M$.

Since $T_{k}^{1} M$ is the Whitney sum of $k$ copies of $T M$ we deduce that a $k$-vector field $\mathbf{X}$ defines $k$ vector fields $\left\{X_{1}, \ldots, X_{k}\right\}$ on $M$ by projecting $\mathbf{X}$ onto every factor. We shall identify $\mathbf{X}$ with the $k$-tuple $\left(X_{1}, \ldots, X_{k}\right)$.

Definition II.2: An integral section of the $k$-vector field $\left(X_{1}, \ldots, X_{k}\right)$ passing through a point $x \in M$ is a map $\sigma: U_{0} \subset \mathbf{R}^{k} \rightarrow M$, defined on some neighborhood $U_{0}$ of $0 \in \mathbf{R}^{\mathbf{k}}$, and satisfying

$$
\sigma(0)=x, \quad d \sigma(t)\left(\left.\frac{\partial}{\partial t^{A}}\right|_{t}\right)=X_{A}(\sigma(t)), \quad 1 \leqslant A \leqslant k, \quad \text { for all } t \in U_{0} .
$$

We say that a $k$-vector field $\left(X_{1}, \ldots, X_{k}\right)$ on $M$ is integrable if there is an integral section passing through each point of $M$.

Remark II.3: Let us consider the trivial bundle $\pi: E=R^{k} \times M \rightarrow R^{k}$. A jet field $\gamma$ on $\pi$ (see Ref. 5) is a section of the projection $\pi_{1,0}: J^{1} \pi \equiv \mathbf{R}^{k} \times T_{k}^{1} M \rightarrow E \equiv \mathbf{R}^{k} \times M$. We identify each $k$-vector field $\mathbf{X}$ on $M$ with the jet field $\gamma=\left(i d_{\mathbf{R}^{k}}, \mathbf{X}\right)$, that is $\gamma(t, x)=\left(t, X_{1}(x), \ldots, X_{k}(x)\right)$. The integral sections of the jet field $\gamma$ (see Ref. 5) correspond to the solutions of the $k$-vector field $\mathbf{X}$ (see Ref. 27). For this reason the solutions of $\mathbf{X}$ are called its integral sections.

Let us remark that if $\sigma$ is an integral section of a $k$-vector field $\left(X_{1}, \ldots, X_{k}\right)$ then each curve on $M$ defined by $\sigma_{A}=\sigma\left(s \mathbf{r}_{\mathbf{A}}\right)$ is an integral curve of the vector field $X_{A}$ on $M$.

Howewer, given $k$ integral curves of $X_{1}, \ldots, X_{k}$, respectively, it is not possible in general to reconstruct an integral section of $\left(X_{1}, \ldots, X_{k}\right)$.

Proposition II.4: Assume that the vector fields $\left\{X_{1}, \ldots, X_{k}\right\}$ on $M$ are linearly independent. Then, the $k$-vector field $\left(X_{1}, \ldots, X_{k}\right)$ is integrable if and only if the distribution generated by $\left\{X_{1}, \ldots, X_{k}\right\}$ is integrable.

\section{THE CANONICAL STABLE $k$-TANGENT STRUCTURE}

Let $Q$ be a manifold of dimension $n$ with local coordinates $\left(q^{i}\right)$ and let $\left(q^{i}, v_{A}^{i}\right)$ be the induced coordinates on $T_{k}^{1} Q$. Let $F$ be a tensor field of type $(1,1)$ on $Q$ such that $F=\Sigma_{i, j} F_{j}^{i}\left(\partial / \partial q^{i}\right)$ $\otimes d q^{j}$. Then the $A$-lift $F^{A}$ of $F$ to $T_{k}^{1} Q$ is the (1,1)-tensor field with local expression:

$$
F^{A}=\sum_{i, j} F_{j}^{i}\left(\frac{\partial}{\partial v_{A}^{i}}\right) \otimes d q^{j}
$$

(see Ref. 26 for further details about the intrinsic construction). If $I_{M}=\Sigma_{i}\left(\partial / \partial q^{i}\right) \otimes d q^{i}$ is the identity tensor field on $M$ then for each $A \in\{1,2, \ldots k\}$, its $A$-lifting defines the tensor field $\widetilde{J}_{A}$ $=I^{A}$ locally given by 


$$
\widetilde{J}_{A}=\sum_{i} \frac{\partial}{\partial v_{A}^{i}} \otimes d q^{i}
$$

The family $\left(\widetilde{J}_{1}, \ldots, \widetilde{J}_{k}\right)$ is called the canonical k-tangent structure of $T_{k}^{1} M$ (see Ref. 28).

Let $J^{1}\left(\mathbf{R}^{\mathbf{k}}, Q\right)$ be the $k+(k+1) n$-dimensional manifold of one jets from $\mathbf{R}^{\mathbf{k}}$ to $Q$, with elements denoted by $j_{t}^{1} \phi$. This manifold can be identified with the manifold $J^{1} \pi$ of one jets of sections of the trivial bundle $\pi: \mathbf{R}^{\mathbf{k}} \times Q \rightarrow \mathbf{R}^{\mathbf{k}}$.

$J^{1} \pi$ is diffeomorphic to $\mathbf{R}^{\mathbf{k}} \times T_{k}^{1} Q$ by composing the following diffeomorphisms:

$$
\begin{gathered}
J^{1} \pi \rightarrow \mathbf{R}^{\mathbf{k}} \times J_{0}^{1}\left(\mathbf{R}^{\mathbf{k}}, Q\right) \rightarrow \mathbf{R}^{\mathbf{k}} \times T_{k}^{1} Q, \\
j_{t}^{1} \phi \rightarrow\left(t, j_{0}^{1} \phi_{t}\right) \rightarrow\left(t, v_{1}, \ldots, v_{k}\right),
\end{gathered}
$$

where

$$
\phi_{t}\left(t^{\prime}\right)=\phi\left(t+t^{\prime}\right), \quad t^{\prime} \in \mathbf{R}^{\mathbf{k}}, \quad v_{A}=\frac{d}{d s}\left(\phi_{t}^{A}\right)_{\mid s=0}, \quad s \in \mathbf{R}, \quad 1 \leqslant A \leqslant k,
$$

and $\phi_{t}^{A}(s)=\phi_{t}\left(s \mathbf{r}_{\mathbf{A}}\right)$.

Let $\tau: \mathbf{R}^{\mathbf{k}} \times T_{k}^{1} Q \rightarrow Q$ be the canonical projection. If $\left(q^{i}\right)$ are local coordinates on $U \subseteq Q$ then the induced local coordinates $\left(t^{A}, q^{i}, v_{A}^{i}\right), 1 \leqslant i \leqslant n, 1 \leqslant A \leqslant k$, on $\tau^{-1}(U) \equiv \mathbf{R}^{\mathbf{k}} \times T_{k}^{1} U$ are given by

$$
t^{A}\left(j_{t}^{1} \phi\right)=t^{A}, \quad q^{i}\left(j_{t}^{1} \sigma\right)=q^{i}\left(\phi_{t}(0)\right), \quad v_{A}^{i}\left(j_{t}^{1} \phi\right)=\frac{d}{d s}\left(q^{i} \circ \phi_{t}^{A}\right)_{\mid s=0}=v_{A}\left(q^{i}\right) .
$$

Therefore we obtain $\tau\left(t^{A}, q^{i}, v_{A}^{i}\right)=\left(q^{i}\right)$.

On $\mathbf{R}^{\mathbf{k}} \times T_{k}^{1} Q$ there exist a family of $k$ tensor fields $J_{A}$ of type $(1,1)$ defined by

$$
J_{A}=\frac{\partial}{\partial t^{A}} \otimes d t^{A}+\widetilde{J}_{A}=\frac{\partial}{\partial t^{A}} \otimes d t^{A}+\sum_{i=1}^{n} \frac{\partial}{\partial v_{A}^{i}} \otimes d q^{i}, \quad 1 \leqslant A \leqslant k,
$$

where we have transported the canonical $k$-tangent structure $\left(\widetilde{J}_{1}, \ldots, \widetilde{J}_{k}\right)$ of $T_{k}^{1} Q$ to $\mathbf{R}^{\mathbf{k}} \times T_{k}^{1} Q$.

If we set $\bar{\eta}_{A}=d t^{A}$ and $\xi_{A}=\partial / \partial t^{A}$, then the family $\left(J_{A}, \bar{\eta}_{A}, \xi_{A} ; 1 \leqslant A \leqslant k\right)$ is called the canonical stable k-tangent structure on $\mathbf{R}^{\mathbf{k}} \times T_{k}^{1} Q$, and $\mathbf{R}^{\mathbf{k}} \times T_{k}^{1} Q$ is the stable $k$-tangent bundle of $Q$.

\section{SECOND ORDER PARTIAL DIFFERENTIAL EQUATIONS}

In this section we characterize the $k$-vector fields on $\mathbf{R}^{k} \times T_{k}^{1} Q$ such that their integral sections are canonical prolongations of maps from $\mathbf{R}^{k}$ to $Q$.

Let $C$ be the canonical vector field of the vector bundle $\pi_{1,0}: \mathbf{R}^{k} \times T_{k}^{1} Q \rightarrow \mathbf{R}^{\mathbf{k}} \times Q$. This vector field $C$ is the infinitesimal generator of the following flow:

$$
\begin{aligned}
& \mathbf{R} \times\left(\mathbf{R}^{k} \times T_{k}^{1} Q\right) \rightarrow \mathbf{R}^{k} \times T_{k}^{1} Q \\
& \left(s,\left(t, q^{i}, v_{A}^{i}\right)\right) \rightarrow\left(t, q^{i}, e^{s} v_{A}^{i}\right),
\end{aligned}
$$

and in local coordinates it has the form

$$
C=\sum_{i, A} v_{A}^{i} \frac{\partial}{\partial v_{A}^{i}} .
$$

$C$ is a sum of vector fields, 


$$
C=\sum_{A=1}^{k} C_{A}
$$

where each $C_{A}$ is the infinitesimal generator of the following flow:

$$
\begin{gathered}
\mathbf{R} \times\left(\mathbf{R}^{k} \times T_{k}^{1} Q\right) \rightarrow \mathbf{R}^{k} \times T_{k}^{1} Q \\
\left(s,\left(t, q^{i}, v_{A}^{i}\right)\right) \rightarrow\left(t, q^{i}, v_{1}^{i}, \ldots, v_{A-1}^{i}, e^{s} v_{A}^{i}, v_{A+1}^{i}, \ldots, v_{k}^{i}\right) .
\end{gathered}
$$

In local coordinates they have the form

$$
C_{A}=\sum_{i=1}^{n} v_{A}^{i} \frac{\partial}{\partial v_{A}^{i}}, \quad 1 \leqslant A \leqslant k .
$$

Let us consider on $\mathbf{R}^{k} \times T_{k}^{1} Q$ the tensor fields $\hat{J}_{1}, \ldots, \hat{J}_{k}$ of type $(1,1)$, defined as follows:

$$
\hat{J}_{A}=\widetilde{J}_{A}-C_{A} \otimes d t^{A}, \quad 1 \leqslant A \leqslant k .
$$

Definition IV.1: A k-vector field $\zeta=\left(\zeta_{1}, \ldots, \zeta_{k}\right)$ on $\mathbf{R}^{k} \times T_{k}^{1} Q$ is said to be a second order partial differential equation (SOPDE for short) if

$$
\hat{J}_{A}\left(\zeta_{A}\right)=0, \quad \bar{\eta}_{A}\left(\zeta_{B}\right)=\delta_{A B},
$$

for all $1 \leqslant A, B \leqslant k$.

From a direct computation in local coordinates we obtain that the local expression of a SOPDE $\zeta=\left(\zeta_{1}, \ldots, \zeta_{k}\right)$ on $\mathbf{R}^{k} \times T_{k}^{1} Q$ is

$$
\zeta_{A}=\frac{\partial}{\partial t^{A}}+v_{A}^{i} \frac{\partial}{\partial q^{i}}+\left(\zeta_{A}\right)_{B}^{i} \frac{\partial}{\partial v_{B}^{i}}, \quad 1 \leqslant A \leqslant k,
$$

where $\left(\zeta_{A}\right)_{B}^{i}$ are functions on $\mathbf{R}^{k} \times T_{k}^{1} Q$.

As a direct consequence of the above local expressions, we deduce that the familiy of vector fields $\left\{\zeta_{1}, \ldots, \zeta_{k}\right\}$ are linearly independent.

Definition IV.2: Let $\phi: \mathbf{R}^{k} \rightarrow Q$ be a map, we define the first prolongation $\phi^{(1)}$ of $\phi$ as the map

$$
\begin{gathered}
\phi^{(1)}: \mathbf{R}^{k} \rightarrow J^{1} \pi \equiv \mathbf{R}^{k} \times T_{k}^{1} Q, \\
t \rightarrow j_{t}^{1} \phi \equiv\left(t, j_{0}^{1} \phi_{t}\right) .
\end{gathered}
$$

In local coordinates:

$$
\phi^{(1)}\left(t^{1}, \ldots, t^{k}\right)=\left(t^{1}, \ldots, t^{k}, \phi^{i}\left(t^{1}, \ldots, t^{k}\right), \frac{\partial \phi^{i}}{\partial t^{A}}\left(t^{1}, \ldots, t^{k}\right)\right), \quad 1 \leqslant A \leqslant k, 1 \leqslant i \leqslant n .
$$

From (5) it follows that an integral section $\sigma$ of a SOPDE $\zeta$ is the first prolongation $\phi^{(1)}$ of a map $\phi$ from $\mathbf{R}^{\mathbf{k}}$ to $Q$.

Definition IV.3: $\phi: \mathbf{R}^{k} \rightarrow Q$ is called a solution of the SOPDE $\zeta$ on $\mathbf{R}^{k} \times T_{k}^{1} Q$ if the first prolongation $\phi^{(1)}$ of $\phi$ is an integral section of $\zeta$.

From (5) and (6) we have the following.

Proposition IV.4: $\phi: \mathbf{R}^{k} \rightarrow Q$ is a solution of the SOPDE $\zeta=\left(\zeta_{1}, \ldots, \zeta_{k}\right)$, locally given by (5), if and only if

$$
\frac{\partial \phi^{i}}{\partial t^{A}}=v_{A}^{i}, \quad \frac{\partial^{2} \phi^{i}}{\partial t^{A} \partial t^{B}}=\left(\zeta_{A}\right)_{B}^{i} .
$$


Remark IV.5: Let us consider the trivial bundles $\pi: E=R^{k} \times Q \rightarrow R^{k}$ and $\pi_{1}: \mathbf{R}^{\mathbf{k}} \times T_{k}^{1} Q \rightarrow \mathbf{R}^{\mathbf{k}}$. We identify each SOPDE $\left(\zeta_{1}, \ldots, \zeta_{k}\right)$ with the following semi-holonomic second order jet field:

$$
\begin{gathered}
J^{1} \pi \equiv \mathbf{R}^{\mathbf{k}} \times T_{k}^{1} Q \rightarrow J^{1} \pi_{1} \equiv \mathbf{R}^{\mathbf{k}} \times T_{k}^{1}\left(T_{k}^{1} Q\right), \\
\left(t^{A}, q^{i}, v_{A}^{i}\right) \rightarrow\left(t^{A}, q^{i}, v_{A}^{i}, v_{A}^{i},\left(\zeta_{A}\right)_{B}^{i}\right) .
\end{gathered}
$$

If the SOPDE $\zeta$ on $\mathbf{R}^{k} \times T_{k}^{1} Q$ is integrable, then its integral sections are canonical prolongations of maps from $\mathbf{R}^{\mathbf{k}}$ to $Q$ and then $\zeta$ defines a second order jet field $\Gamma$ on $\pi$ whose coordinate representation of the corresponding connection $\widetilde{\Gamma}$ is

$$
\widetilde{\Gamma}=d t^{A} \otimes\left(\frac{\partial}{\partial t^{A}}+v_{A}^{i} \frac{\partial}{\partial q^{i}}+\left(\zeta_{A}\right)_{B}^{i} \frac{\partial}{\partial v_{B}^{i}}\right)
$$

since $\left(\zeta_{A}\right)_{B}^{i}=\left(\zeta_{B}\right)_{A}^{i}$ (see Ref. 5).

The integrability of the SOPDE is equivalent to the condition given by $\mathcal{R}=0$, where $\mathcal{R}$ is the curvature tensor of the above connection (see Ref. 13 and 5).

\section{V. $k$-COSYMPLECTIC STRUCTURES}

The keystone of the nonautonomous Lagrangian formalism of classical mechanics is the cosymplectic structure on the phase space $\mathbf{R} \times T Q$. For field theory we shall show that this statement remains true, provided the two- forms that define the cosymplectic structure are replaced by two vector valued forms.

Let $J^{1}\left(Q, \mathbf{R}^{\mathbf{k}}\right)_{0}$ be the manifold of one jets of maps from $Q$ to $\mathbf{R}^{\mathbf{k}}$ with target at $0 \in \mathbf{R}^{\mathbf{k}}$.

The manifold $J^{1}\left(Q, \mathbf{R}^{\mathbf{k}}\right)_{0}$ can be canonically identified with the Whitney $\operatorname{sum}\left(T_{k}^{1}\right)^{*} Q$ of $k$ copies of $T^{*} Q$, that is

$$
\begin{gathered}
J^{1}\left(Q, \mathbf{R}^{\mathbf{k}}\right)_{0} \rightarrow\left(T_{k}^{1}\right)^{*} Q=T^{*} Q \oplus \cdots \oplus T^{*} Q, \\
j_{q, 0}^{1} \sigma \equiv\left(p_{q}^{1}, \ldots, p_{q}^{k}\right),
\end{gathered}
$$

where $p_{q}^{A}=d\left(\pi_{A^{\circ}} \sigma\right)(q)$ being $\pi_{A}: \mathbf{R}^{\mathbf{k}} \rightarrow \mathbf{R}$ the canonical projection.

Let $J^{1}\left(Q, \mathbf{R}^{\mathbf{k}}\right)$ be the $k+(k+1) n$-dimensional manifold of one jets from $Q$ to $\mathbf{R}^{\mathbf{k}}$, with elements denoted by $j_{q, t}^{1} \sigma$. We recall that one jets of mappings from $Q$ to $\mathbf{R}^{\mathbf{k}}$ can be identified with the manifold $J^{1} \rho$ of one jets of sections of the trivial bundle $\rho: \mathbf{R}^{\mathbf{k}} \times Q \rightarrow Q$.

$J^{1} \rho$ is diffeomorphic to $\mathbf{R}^{\mathbf{k}} \times\left(T_{k}^{1}\right)^{*} Q$, via the diffeomorphism given by

$$
j_{q}^{1} \alpha \in J^{1} \rho \rightarrow\left(\sigma(q), j_{q, 0}^{1} \sigma_{q}\right) \in \mathbf{R}^{\mathbf{k}} \times\left(T_{k}^{1}\right) * Q,
$$

where $\sigma_{q}(\widetilde{q})=\sigma(\widetilde{q})-\sigma(q)$ and $\widetilde{q}$ denotes an arbitrary point in $Q$.

Let us denote by $\tau^{*}: \mathbf{R}^{\mathbf{k}} \times\left(T_{k}^{1}\right)^{*} Q \rightarrow Q$ the canonical projection. If $\left(q^{i}\right)$ are local coordinates on $U \subseteq Q$ then the induced local coordinates $\left(t^{A}, q^{i}, p_{i}^{A}\right), 1 \leqslant i \leqslant n, 1 \leqslant A \leqslant k$, on $\left(\tau^{*}\right)^{-1}(U)$ $\equiv \mathbf{R}^{\mathbf{k}} \times\left(T_{k}^{1}\right) * U$ are given by

$$
t^{A}\left(j_{q}^{1} \sigma\right)=t^{A}(\sigma(q)), \quad q^{i}\left(j_{q}^{1} \sigma\right)=q^{i}(q), \quad p_{i}^{A}\left(j_{q}^{1} \sigma\right)=d\left(\sigma_{q}^{A}\right)(q)\left(\frac{\partial}{\left.\partial q^{i}\right|_{q}}\right),
$$

where $\sigma_{q}^{A}=\pi_{A^{\circ}} \sigma_{q}$.

An $\mathbf{R}^{\mathbf{k}}$-valued one-form $\eta_{0}$ and a $\mathbf{R}^{\mathbf{k}}$-valued two-form $\omega_{0}$ on $\mathbf{R}^{\mathbf{k}} \times\left(T_{k}^{1}\right)^{*} Q$ are defined by

$$
\eta_{0}=\sum_{A=1}^{m}\left(\eta_{0}\right)_{A} \mathbf{r}_{\mathbf{A}}=\sum_{A=1}^{k}\left(\left(\pi_{A}^{1}\right)^{*} d t\right) \mathbf{r}_{\mathrm{A}}, \quad \omega_{0}=\sum_{A=1}^{k}\left(\omega_{0}\right)_{A} \mathbf{r}_{\mathbf{A}}=\sum_{A=1}^{m}\left(\pi_{A}^{2}\right)^{*}\left(\omega_{Q}\right) \mathbf{r}_{\mathrm{A}},
$$


where $\pi_{A}^{1}: \mathbf{R}^{\mathbf{k}} \times\left(T_{k}^{1}\right)^{*} Q \rightarrow \mathbf{R}$ and $\pi_{A}^{2}: \mathbf{R}^{\mathbf{k}} \times\left(T_{k}^{1}\right)^{*} Q \rightarrow T^{*} Q$ are the projections defined by

$$
\pi_{A}^{1}\left(\left(t^{B}\right),\left(p^{B}\right)\right)=t^{A}, \quad \pi_{A}^{2}\left(\left(t^{B}\right),\left(p^{B}\right)\right)=p^{A},
$$

and $\omega_{Q}$ is the canonical symplectic form on $T^{*} Q$.

In local coordinates we have

$$
\left(\eta_{0}\right)_{A}=d t^{A}, \quad\left(\omega_{0}\right)_{A}=\sum_{i=1}^{n} d q^{i} \wedge d p_{i}^{A} \quad 1 \leqslant A \leqslant k .
$$

Moreover, let be $V=\operatorname{ker} T \mu^{*}$, where $\mu^{*}: \mathbf{R}^{\mathbf{k}} \times\left(T_{k}^{1}\right)^{*} Q \rightarrow \mathbf{R}^{\mathbf{k}} \times Q$. A simple inspection in local coordinates shows that the forms $\left(\eta_{0}\right)_{A}$ and $\left(\omega_{0}\right)_{A}$ are closed and the following relations hold:

$$
\left(\eta_{0}\right)_{1} \wedge \cdots \wedge\left(\eta_{0}\right)_{k} \neq 0,\left(\eta_{0}\right)_{A_{J V}}=0, \omega_{A_{J V} \times V}=0
$$

(ii) $\quad\left(\cap_{A=1}^{n} \operatorname{ker}\left(\eta_{0}\right)_{A}\right) \cap\left(\cap_{A=1}^{n} \operatorname{ker}\left(\omega_{0}\right)_{A}\right)=\{0\}, \operatorname{dim}\left(\cap_{A=1}^{n} \operatorname{ker}\left(\omega_{0}\right)_{A}\right)=k$.

Inspired in the above geometrical model we have introduced in Ref. 22 the following definition.

Definition V.1: Let $M$ be a differentiable manifold of dimension $(k+1) n+k$. A family $\left(\eta_{A}, \omega_{A}, V ; 1 \leqslant A \leqslant k\right)$, where each $\eta_{A}$ is a closed 1-form, each $\omega_{A}$ is a closed 2 -form and $V$ is an $n k$-dimensional integrable distribution on $M$, such that

(i) $\eta_{1} \wedge \cdots \wedge \eta_{k} \neq 0, \eta_{A_{J V}}=0, \omega_{A_{J V \times V}}=0$,

(ii) $\left(\cap_{A=1}^{k} \operatorname{ker} \eta_{A}\right) \cap\left(\cap_{A=1}^{k} \operatorname{ker} \omega_{A}\right)=\{0\}, \operatorname{dim}\left(\cap_{A=1}^{k} \operatorname{ker} \omega_{A}\right)=k$,

is called a k-cosymplectic structure, and the manifold $M$ a k-cosymplectic manifold.

The canonical model for these geometrical structures is $\left(\mathbf{R}^{\mathbf{k}} \times\left(T_{k}^{1}\right)^{*} Q, \eta_{0}, \omega_{0}, V\right)$.

For any $k$-cosymplectic structure $\left(\eta_{A}, \omega_{A}, V\right)$ on $M$, there exists a family of $k$ vector fields $\left(\xi_{1}, \ldots, \xi_{k}\right)$ characterized by the conditions

$$
\eta_{A}\left(\xi_{B}\right)=\delta_{A B}, \quad \iota_{\xi_{B}} \omega_{A}=0
$$

for all $1 \leqslant A, B \leqslant k$. These vector fields are called the Reeb vector fields associated to the $k$-cosymplectic structure.

If $(M, \eta, \omega, V)$ is a $k$-cosymplectic manifold we can define the vector bundle morphism,

$$
\begin{gathered}
\Omega^{\sharp}: T_{k}^{1} M \rightarrow T^{*} M \\
\left(X_{1}, \ldots, X_{k}\right) \rightarrow \Omega^{\sharp}\left(X_{1}, \ldots, X_{k}\right)=\sum_{A=1}^{k} \iota_{X_{A}} \omega_{A}+\eta_{A}\left(X_{A}\right) \eta_{A} .
\end{gathered}
$$

Remark V.2: If $k=1$ then $\Omega^{\#}$ is defined from $T M$ onto $T^{*} M$ and it is in fact the isomorphism $\chi_{\eta, \omega}$ defined on the cosymplectic manifold $(M, \eta, \omega)$ by (see Ref. 23,24$)$

$$
\chi_{\eta, \omega}(X)=\iota_{X} \omega+\eta(X) \eta
$$

Let $\left(M, \eta_{A}, \omega_{A}, V\right)$ be a $k$-cosymplectic manifold, $H: M \rightarrow \mathbf{R}$ a Hamiltonian function and $\xi_{A}$ are the Reeb vector fields determined by $\left(\eta_{A}, \omega_{A}, V\right)$. In Ref. 22 we have proved that if an integrable $k$-vector field $\mathbf{X}=\left(X_{1}, \ldots, X_{k}\right)$ satisfies the equations

$$
\begin{gathered}
\eta_{A}\left(X_{B}\right)=\delta_{A B}, \quad \forall A, B, \\
\Omega^{\#}\left(X_{1}, \ldots, X_{k}\right)=d H+\sum_{A=1}^{k}\left(1-\xi_{A}(H)\right) \eta_{A},
\end{gathered}
$$


then its integral sections $\phi$ are solutions of the Hamiltonian field equations corresponding to $H$ :

$$
\frac{\partial H}{\partial x^{i}}=-\sum_{A=1}^{k} \frac{\partial \phi_{i}^{A}}{\partial s^{A}}, \quad \frac{\partial H}{\partial x_{i}^{A}}=\frac{\partial \phi^{i}}{\partial s^{A}} .
$$

The existence of canonical coordinates $\left(s^{A}, x^{i}, x_{i}^{A}\right)$ is ensured by the corresponding Darboux theorem (see Ref. 22).

\section{THE LAGRANGIAN FORMALISM}

Given a Lagrangian function of the form $L=L\left(t^{A}, q^{i}, v_{A}^{i}\right)$ one obtains, by using a variational principle, the Euler-Lagrange equations for $L$ :

$$
\sum_{A=1}^{k} \frac{d}{d t^{A}}\left(\frac{\partial L}{\partial v_{A}^{i}}\right)-\frac{\partial L}{\partial q^{i}}=0, \quad v_{A}^{i}=\frac{\partial q^{i}}{\partial t^{A}} .
$$

First of all, one realizes that such a $L$ can be considered as a function $L: \mathbf{R}^{\mathbf{k}} \times T_{k}^{1} Q \rightarrow \mathbf{R}$.

In this section we shall give a geometrical description of the above equations (11) using a $k$-cosymplectic structure on $\mathbf{R}^{\mathbf{k}} \times T_{k}^{1} Q$ associated to the regular Lagrangian $L$. This $k$-cosymplectic structure shall be constructed using the canonical stable $k$-tangent structure of $\mathbf{R}^{\mathbf{k}} \times T_{k}^{1} Q$.

For each $1 \leqslant A \leqslant k$, we define the following.

- The vertical derivation $\iota_{J_{A}}$ of forms on $\mathbf{R}^{\mathbf{k}} \times T_{k}^{1} Q$ by

$$
\iota_{J_{A}} f=0, \quad\left(\iota_{J_{A}} \alpha\right)\left(X_{1}, \ldots, X_{p}\right)=\sum_{j=1}^{p} \alpha\left(X_{1}, \ldots, J_{A} X_{j}, \ldots, X_{p}\right),
$$

for any function $f$ and any $p$-form $\alpha$ on $\mathbf{R}^{\mathbf{k}} \times T_{k}^{1} Q$;

- the vertical differentation $d_{J_{A}}$ of forms on $\mathbf{R}^{\mathbf{k}} \times T_{k}^{1} Q$ by

$$
d_{J_{A}}=\left[\iota_{J_{A}}, d\right]=\iota_{J_{A}}{ }^{\circ} d-d^{\circ} \iota_{J_{A}},
$$

where $d$ denotes the usual exterior differentation.

Let us consider the 1 -forms,

$$
\left(\beta_{L}\right)_{A}=d_{J_{A}} L-\xi_{A}(L) \bar{\eta}_{A}, \quad 1 \leqslant A \leqslant k .
$$

In bundle coordinates $\left(t^{A}, q^{i}, v_{A}^{i}\right)$ we have

$$
\left(\beta_{L}\right)_{A}=\sum_{i=1}^{n} \frac{\partial L}{\partial v_{A}^{i}} d q^{i}, \quad 1 \leqslant i \leqslant k .
$$

Definition VI.1: A Lagrangian $L$ is called regular if and only if the Hessian matrix,

$$
\left(\frac{\partial^{2} L}{\partial v_{A}^{i} \partial v_{B}^{j}}\right)
$$

is nonsingular.

Now, we introduce the following 2-forms:

$$
\left(\omega_{L}\right)_{A}=-d\left(\beta_{L}\right)_{A}, \quad 1 \leqslant A \leqslant k .
$$

Proposition VI.2: Let $L: \mathbf{R}^{\mathbf{k}} \times T_{k}^{1} Q \rightarrow \mathbf{R}$ be a regular Lagrangian, and $V_{0}$ the vertical distribution of the bundle $\pi_{1,0}: \mathbf{R}^{\mathbf{k}} \times T_{k}^{1} Q \rightarrow \mathbf{R}^{\mathbf{k}} \times Q$. Then, $L$ is regular if and only if $\left(\mathbf{R}^{\mathbf{k}} \times T_{k}^{1} Q, \bar{\eta}_{A},\left(\omega_{L}\right)_{A}, V_{0}\right)$ is a k-cosymplectic manifold. 
Proof: Assume that $L$ is a regular Lagrangian. We shall prove that $\left(\mathbf{R}^{\mathbf{k}} \times T_{k}^{1} Q, \bar{\eta}_{A},\left(\omega_{L}\right)_{A}, V_{0}\right)$ is a $k$-cosymplectic manifold.

Conditions (i) are rather obvious. Now, we shall prove condition (ii) in Definition V.1. Let $X$ be a vector field with the local expression

$$
X=X^{A} \frac{\partial}{\partial t^{A}}+X^{i} \frac{\partial}{\partial q^{i}}+X_{A}^{i} \frac{\partial}{\partial v_{A}^{i}},
$$

such that

$$
\iota_{X}\left(\omega_{L}\right)_{A}=0, \text { and } \bar{\eta}_{A}(X)=0, \text { for all } 1 \leqslant A \leqslant k .
$$

The second condition implies that $X^{A}=0$, for all $A$, and then the first condition yields

$$
X^{i} \frac{\partial^{2} L}{\partial v_{A}^{i} \partial v_{B}^{j}}=0
$$

and

$$
X^{i} \frac{\partial^{2} L}{\partial v_{A}^{i} \partial q^{j}}-X^{i} \frac{\partial^{2} L}{\partial v_{A}^{j} \partial q^{i}}-X_{B}^{i} \frac{\partial^{2} L}{\partial v_{A}^{j} \partial v_{B}^{i}}=0 .
$$

Since $L$ is regular, from (14) we deduce that $X^{i}=0$, for all $i$, so that (15) implies $X_{A}^{i}=0$, for all $i$ and $A$. Therefore, we conclude that $X=0$.

Next, we shall prove the second part of condition (ii) in Definition V.1. Let $Y$ be a vector field with the local expression

$$
Y=Y^{A} \frac{\partial}{\partial t^{A}}+Y^{i} \frac{\partial}{\partial q^{i}}+Y_{A}^{i} \frac{\partial}{\partial v_{A}^{i}},
$$

such that

$$
\iota_{X}\left(\omega_{L}\right)_{A}=0, \text { for all } 1 \leqslant A \leqslant k .
$$

We then have

$$
Y^{j} \frac{\partial^{2} L}{\partial v_{A}^{i} \partial v_{B}^{j}}=0
$$

and

$$
-Y^{B} \frac{\partial^{2} L}{\partial v_{A}^{i} \partial t^{B}}+Y^{j} \frac{\partial^{2} L}{\partial v_{A}^{j} \partial q^{i}}-Y^{j} \frac{\partial^{2} L}{\partial v_{A}^{i} \partial q^{j}}-Y_{B}^{j} \frac{\partial^{2} L}{\partial v_{A}^{i} \partial v_{B}^{j}}=0 .
$$

From (16) and the regularity of $L$ we deduce that $Y^{j}=0$, for all $j$, so that (17) reduces to

$$
-Y^{B} \frac{\partial^{2} L}{\partial v_{A}^{i} \partial t^{B}}-Y_{B}^{j} \frac{\partial^{2} L}{\partial v_{A}^{i} \partial v_{B}^{j}}=0 .
$$

Since the matrix $\left(\partial^{2} L / \partial v_{A}^{i} \partial v_{B}^{j}\right)$ is regular, (18) implies that

$$
Y_{B}^{j}=\mathcal{A}_{B D}^{j} Y^{D},
$$

for some matrix $\mathcal{A}_{B D}^{j}$. Therefore, we get 


$$
Y=Y^{B}\left(\frac{\partial}{\partial t^{B}}+\mathcal{A}_{C B}^{j} \frac{\partial}{\partial v_{C}^{j}}\right)
$$

Thus, $\cap_{A=1}^{n} \operatorname{ker}\left(\omega_{L}\right)_{A}$ is locally spanned by the family of $k$ independente local vector fields $\left\{\partial / \partial t^{B}+\mathcal{A}_{C B}^{j}\left(\partial / \partial v_{C}^{j}\right)\right\}$.

Then we have proved that $\left(\mathbf{R}^{\mathbf{k}} \times T_{k}^{1} Q,\left(\eta_{0}\right)_{A},\left(\omega_{L}\right)_{A}, V_{0}\right)$ is a $k$-cosymplectic manifold.

The converse is proved by reversing the above arguments.

Let $L: \mathbf{R}^{\mathbf{k}} \times T_{k}^{1} Q \rightarrow \mathbf{R}$ be a regular Lagrangian and $\left(\bar{\eta}_{A},\left(\omega_{L}\right)_{A}, V_{0}\right)$ the associated $k$-cosymplectic structure on $\mathbf{R}^{\mathbf{k}} \times T_{k}^{1} Q$.

The equations,

$$
\bar{\eta}_{A}\left(\left(\xi_{L}\right)_{B}\right)=\delta_{B}^{A}, \quad \iota_{\left(\xi_{L}\right)_{A}}\left(\omega_{L}\right)_{B}=0, \quad 1 \leqslant A, B \leqslant k,
$$

define the Reeb vector fields $\left\{\left(\xi_{L}\right)_{1}, \ldots,\left(\xi_{L}\right)_{k}\right\}$ on $\mathbf{R}^{\mathbf{k}} \times T_{k}^{1} Q$ which are locally given by

$$
\left(\xi_{L}\right)_{A}=\frac{\partial}{\partial t^{A}}+\left(\left(\xi_{L}\right)_{A}\right)_{B}^{i} \frac{\partial}{\partial v_{B}^{i}},
$$

where the functions $\left(\left(\xi_{L}\right)_{A}\right)_{B}^{i}$ satisfy

$$
\frac{\partial^{2} L}{\partial t^{A} \partial v_{C}^{j}}+\frac{\partial^{2} L}{\partial v_{B}^{i} \partial v_{C}^{j}}\left(\left(\xi_{L}\right)_{A}\right)_{B}^{i}=0
$$

for all $1 \leqslant A, B, C \leqslant k$ and $1 \leqslant i, j \leqslant n$.

Since $L$ is regular, from the local conditions (21) we can define, in a neighborhood of each point of $\mathbf{R}^{\mathbf{k}} \times T_{k}^{1} Q$, a $k$-vector field that satisfies (19). Next, one can construct a global $k$-vector field $\xi_{L}$, which is a solution of (19), by using a partition of unity.

Let $L$ be a regular Lagrangian and let $\Omega_{L}^{\#}$ be the $\#$-morphism defined by the $k$-cosymplectic structure $\left(\bar{\eta}_{A},\left(\omega_{L}\right)_{A}, V_{0}\right)$, as in (9):

$$
\begin{gathered}
\Omega_{\mathrm{L}}^{\#}: T_{k}^{1}\left(\mathbf{R}^{\mathrm{k}} \times T_{k}^{1} Q\right) \rightarrow T^{*}\left(\mathbf{R}^{\mathbf{k}} \times T_{k}^{1} Q\right) \\
\left(X_{1}, \ldots, X_{k}\right) \rightarrow \Omega_{L}^{\#}\left(X_{1}, \ldots, X_{k}\right)=\sum_{A=1}^{k} \iota_{X_{A}}\left(\omega_{L}\right)_{A}+\bar{\eta}_{A}\left(X_{A}\right) \bar{\eta}_{A} .
\end{gathered}
$$

Theorem VI.3: Let $L$ be a regular Lagrangian and let $\mathbf{X}=\left(X_{1}, \ldots, X_{k}\right)$ be a $k$-vector field such that

$$
\begin{gathered}
\bar{\eta}_{A}\left(X_{B}\right)=\delta_{A B}, \quad 1 \leqslant A, B \leqslant k, \\
\Omega_{L}^{\sharp}\left(X_{1}, \ldots, X_{k}\right)=d E_{L}+\sum_{A=1}^{k}\left(1-\left(\xi_{L}\right)_{A}\left(E_{L}\right)\right) \bar{\eta}_{A},
\end{gathered}
$$

where $E_{L}=C(L)-L$. Then $\mathbf{X}=\left(X_{1}, \ldots, X_{k}\right)$ is a SOPDE. In addition, if $\mathbf{X}=\left(X_{1}, \ldots, X_{k}\right)$ is integrable then its solutions satisfy the Euler-Lagrange equations (11).

Proof: It should be noticed that in general Eqs. (23) have not a unique solution. In fact, the solutions of (23) are given by $\left(X_{1}, \ldots, X_{k}\right)+\left(\operatorname{ker} \Omega^{\#}\right)$, where $\left(X_{1}, \ldots, X_{k}\right)$ is a particular solution. Nevertheless, we shall show now that there exist always solutions of equations (23) when $L$ is assumed to be regular. In this case, from (3) and (20) we obtain

$$
\left(\xi_{L}\right)_{A}\left(E_{L}\right)=-\frac{\partial L}{\partial t^{A}} .
$$


Let $\left(X_{1}, \ldots, X_{k}\right)$ be a solution of (23) locally given by

$$
X_{A}\left(t, q^{i}, v_{A}^{i}\right)=\frac{\partial}{\partial t^{A}}+\left(X_{A}\right)^{i} \frac{\partial}{\partial q^{i}}+\left(X_{A}\right)_{B}^{j} \frac{\partial}{\partial v_{B}^{j}} .
$$

Then, from (24) we obtain

$$
\begin{gathered}
\sum_{A, B, j}\left(-\frac{\partial^{2} L}{\partial t^{A} \partial v_{A}^{i}}+\left(X_{A}\right)^{j}\left(\frac{\partial^{2} L}{\partial q^{i} \partial v_{A}^{j}}-\frac{\partial^{2} L}{\partial q^{j} \partial v_{A}^{i}}\right)-\left(X_{A}\right)_{B}^{j} \frac{\partial^{2} L}{\partial v_{B}^{j} \partial v_{A}^{i}}\right)=\sum_{A, j}\left(v_{A}^{j} \frac{\partial^{2} L}{\partial q^{i} \partial v_{A}^{j}}-\frac{\partial L}{\partial q^{i}}\right) \\
\sum_{A, i}\left(X_{A}\right)^{i} \frac{\partial^{2} L}{\partial v_{B}^{j} \partial v_{A}^{i}}=\sum_{A, i} v_{A}^{i} \frac{\partial^{2} L}{\partial v_{B}^{j} \partial v_{A}^{i}}
\end{gathered}
$$

and since $L$ is regular, from (26) we deduce that the solutions of Eqs. (23) are SOPDE's, that is, each $X_{A}$ has the form

$$
X_{A}\left(t, q^{i}, v_{A}^{i}\right)=\frac{\partial}{\partial t^{A}}+v_{A}^{i} \frac{\partial}{\partial q^{i}}+\left(X_{A}\right)_{B}^{j} \frac{\partial}{\partial v_{B}^{j}} .
$$

From (25) we deduce that the functions $\left(X_{A}\right)_{B}^{j}$ satisfy the equations

$$
\sum_{A} \frac{\partial^{2} L}{\partial t^{A} \partial v_{A}^{i}}+\sum_{j, B}\left(v_{B}^{j} \frac{\partial^{2} L}{\partial q^{j} \partial v_{B}^{i}}+\sum_{A}\left(X_{A}\right)_{B}^{j} \frac{\partial^{2} L}{\partial v_{B}^{j} \partial v_{A}^{i}}\right)=\frac{\partial L}{\partial q^{i}}, \quad 1 \leqslant i \leqslant n .
$$

Since $L$ is regular, (28) leads us to define local solutions of (23) in a neigborhood of each point of $\mathbf{R}^{\mathbf{k}} \times T_{k}^{1} Q$. Using a partition of unity one can easily obtain a global solution of (23).

Now, let

$$
\begin{gathered}
\phi: \mathbf{R}^{\mathbf{k}} \rightarrow Q, \\
\left(t^{B}\right) \rightarrow\left(\phi^{i}\left(t^{1}, \ldots, t^{k}\right)\right),
\end{gathered}
$$

be a solution of $\left(X_{1}, \ldots, X_{k}\right)$. From Proposition IV.4 and Eq. (27) we deduce that

$$
\frac{\partial \phi^{i}}{\partial t^{A}}=v_{A}^{i}, \quad \frac{\partial^{2} \phi_{A}^{i}}{\partial t^{A} \partial t^{B}}=\left(X_{A}\right)_{B}^{i}
$$

Replacing in (28) we get

$$
\sum_{A} \frac{\partial^{2} L}{\partial t^{A} \partial v_{A}^{i}}+\sum_{j, B}\left(\frac{\partial \phi^{j}}{\partial t^{B}} \frac{\partial^{2} L}{\partial q^{j} \partial v_{B}^{i}}+\sum_{A} \frac{\partial^{2} \phi^{j}}{\partial t^{A} \partial t^{B}} \frac{\partial^{2} L}{\partial v_{B}^{j} \partial v_{A}^{i}}\right)=\frac{\partial L}{\partial q^{i}}, \quad 1 \leqslant i \leqslant n,
$$

which shows that $\phi$ is a solution of the Euler-Lagrange equations (11).

In conclussion, we can consider Eqs. (23) as a geometric version of the Euler-Lagrange field equations.

Remark VI.4: We have given a geometric version of the Euler-Lagrange equations for a nonautonomous Lagrangian constructing a $k$-cosymplectic structure on $\mathbf{R}^{\mathbf{k}} \times T_{k}^{1} Q$ defined from the Lagrangian and the canonical stable $k$-tangent structure on $\mathbf{R}^{\mathbf{k}} \times T_{k}^{1} Q$. We can also construct this $k$-cosymplectic structure using the Legendre tranformation $\mathcal{F} L$ of $L$ which is the map

$$
\mathcal{F} L: \mathbf{R}^{\mathbf{k}} \times T_{k}^{1} Q \rightarrow \mathbf{R}^{\mathbf{k}} \times\left(T_{k}^{1}\right)^{*} Q,
$$

defined as follows: if $(t, \mathbf{v})=\left(t^{1}, \ldots, t^{k}, v_{1}, \ldots, v_{k}\right) \in \mathbf{R}^{\mathbf{k}} \times\left(T_{k}^{1} Q\right)_{q}$ with $q \in Q$ and $v_{A} \in T_{q} Q$, then 


$$
\mathcal{F} L(t, y)=\left(t^{1}, \ldots, t^{k}, p^{1}, \ldots p^{k}\right) \in \mathbf{R}^{\mathbf{k}} \times\left(T_{k}^{1} Q\right)_{q}^{*}, \quad p^{A} \in T_{q}^{*} Q
$$

is given by

$$
p^{A}\left(v_{q}\right)=\left(\beta_{L}\right)_{A}\left(\overline{v_{q}}\right), \quad 1 \leqslant A \leqslant k,
$$

for any $v_{q} \in T_{q} Q$, where $\overline{v_{q}} \in T_{v}\left(T_{k}^{1} Q\right)$ is any tangent vector such that $d \tau_{Q}(v)\left(\overline{v_{q}}\right)=v_{q}$, with $\tau_{Q}: T_{k}^{1} Q \rightarrow Q$ the canonical projection. In induced coordinates we have

$$
\mathcal{F} L:\left(t^{A}, q^{i}, v_{A}^{i}\right) \rightarrow\left(t^{A}, q^{i}, \frac{\partial L}{\partial v_{A}^{i}}\right) .
$$

The Jacobian matrix of $\mathcal{F} L$ is

$$
\left(\begin{array}{cccccc}
I_{k} & & 0 & 0 & \ldots & 0 \\
0 & & I_{n} & 0 & \ldots & 0 \\
& \mathcal{B} & & & \mathcal{C} &
\end{array}\right),
$$

where $I_{k}$ e $I_{n}$ are the identity matrices of order $k$ and $n$, respectively, $\mathcal{B}$ is a matrix $n k \times(k+n)$ and $\mathcal{C}$ is the matrix

$$
\left(\frac{\partial^{2} L}{\partial v_{A}^{i} \partial v_{B}^{j}}\right)
$$

Now, from (12) and (30) we deduce the following.

Lemma VI.5: We have

$$
\left(\omega_{L}\right)_{A}=\mathcal{F} L^{*}\left(\left(\omega_{0}\right)_{A}\right), \quad \bar{\eta}_{A}=\mathcal{F} L^{*}\left(\left(\eta_{0}\right)_{A}\right),
$$

for all $A$.

Next, from (31) we obtain the following proposition.

Proposition VI.6: The following conditions are equivalent:

(1) $L$ is regular.

(2) $\mathcal{F} L$ is a local diffeomorphism.

(3) $\left(\bar{\eta}_{1}, \ldots, \bar{\eta}_{k},\left(\omega_{L}\right)_{1}, \ldots,\left(\omega_{L}\right)_{k}, V_{0}\right)$ is a k-cosymplectic structure on $\mathbf{R}^{k} \times T_{k}^{1} Q$.

\section{SINGULAR CASE}

When the Lagrangian function $L$ is not regular, the family $\left(\bar{\eta}_{A},\left(\omega_{L}\right)_{A}, V_{0}, 1 \leqslant A \leqslant k\right)$ is no longer a $k$-cosymplectic structure. Even in this case, from (24), we deduce that if $\mathbf{X}$ $=\left(X_{1}, \ldots, X_{k}\right)$ is an integrable SOPDE such that

$$
\begin{gathered}
\bar{\eta}_{A}\left(X_{B}\right)=\delta_{A B}, \\
\Omega_{L}^{\#}\left(X_{1}, \ldots, X_{k}\right)=d E_{L}+\sum_{A=1}^{k}\left(1+\frac{\partial}{\partial t^{A}}(L)\right) \bar{\eta}_{A},
\end{gathered}
$$

then its solutions satisfy the Euler-Lagrange equations.

But we cannot assure that such a solution exists. Therefore we shall develop a constraint algorithm inspired in the well-known one for singular Lagrangians in mechanics in order to obtain a final constraint submanifold where such a solution exists.

We put $P_{1}=\mathbf{R}^{k} \times T_{k}^{1} Q$. Next, let $P_{2}$ be the subset of $P_{1}$ which consists of those points where there exists solution of (32), that is, 


$$
P_{2}=\left\{z \in P_{1} / \exists \mathbf{X} \in\left(T_{k}^{1}\left(\mathbf{R}^{k} \times\left(T_{k}^{1}\right)^{*} Q\right)\right)_{z}\right. \text { satisfying the SOPDE condition and }
$$

being a solution of $(32)\}$.

If $P_{2}$ is a submanifold of $P_{1}$, then there exists a section of $\tau_{\mathbf{R}^{k} \times T_{k}^{1} Q}$ over $P_{2}$. This section is not in general a $k$-vector field on $P_{2}$. To find solutions taking values into $T_{k}^{1} P_{2}$ we construct a new subset $P_{3}$ of $P_{2}$ as follows:

$$
P_{3}=\left\{z \in P_{2} / \exists \mathbf{X} \in\left(T_{k}^{1} P_{2}\right)_{z} \text { satisfying the SOPDE condition and being a solution of (32) }\right\} .
$$

If $P_{3}$ is a submanifold of $P_{2}$, there exists a section of $\tau_{P_{2}}$ over $P_{3}$ which is solution of (23), but that not defines, in general, a $k$-vector field on $P_{3}$.

Proceeding further, we get a family of constraint manifolds,

$$
\cdots \rightarrow P_{3} \rightarrow P_{2} \rightarrow P_{1}=\mathbf{R}^{k} \times T_{k}^{1} Q .
$$

In the most favorable case, this constraint algorithm stabilizes at some step, say $P_{h+1}=P_{h}$ and $\operatorname{dim} P_{h}>0$. In this case, we call $P_{f}=P_{h}$ the final constraint manifold. On $P_{f}$ there exists a SOPDE solution of (32).

Of course, this solution would not be unique as in the regular case. It should be noticed that, in addition, the solutions on $P_{f}$ shall not be in principle integrable. To guarantee the existence of an integrable solution one has to develop an additional constraint algorithm taking the brackets of the vector fields which compose a SOPDE solution. Doing that we shall obtain an integrable SOPDE solution on a (smaller, in general) submanifold $S$ of $P_{f}$; its solutions shall satisfy the Euler-Lagrange equations (11) (see Ref. 13).

\section{ACKNOWLEDGMENTS}

This work has been partially supported through Grants No. DGICYT (Spain) (Project No. PB97-1257), Xunta de Galicia (Project No. XUGA 20703B98) and the Universidade da Coruñ. We acknowledge the referee for many useful remarks and suggestions.

\footnotetext{
${ }^{1}$ M. de León and P. R. Rodrigues, Generalized Classical Mechanics and Field Theory (North-Holland Mathematical Studies, Amsterdam, 1985), p. 112.

${ }^{2}$ E. Binz, J. Śniatycki, and H. Fisher, Geometry of Classical Fields (North-Holland Mathematical Studies, Amsterdam, 1989), p. 154.

${ }^{3}$ G. Sardanashvily, Gauge Theory in Jet Manifolds (Hadronic, Palm Harbor, 1993).

${ }^{4}$ G. Sardanashvily, Generalized Hamiltonian Formalism for Field Theory: Constrained Systems (World Scientific, Singapore, 1995).

${ }^{5}$ D. J. Saunders, The Geometry of Jet Bundles, London Mathematical Society Lecture Notes Series, (Cambridge University Press, Cambridge, 1989), p. 142.

${ }^{6}$ F. Cantrijn, A. Ibort, and M. de León, "On the geometry of multisymplectic manifolds," J. Aust. Math. Soc., Ser. A 66, 303-330 (1999).

${ }^{7}$ F. Cantrijn, A. Ibort, and M. de León, Hamiltonian structures on multisymplectic manifolds, Rend. Sem. Mat. Univ. Pol. Torino 54, 225-236 (1996).

${ }^{8}$ M. Gotay, J. Isenberg, and J. E. Marsden, Momentum Maps and Classical Relativistic Fields, Part I: Covariant Field Theory (1997).

${ }^{9}$ M. Gotay, J. Isenberg, and J. E. Marsden, Momentum Maps and Classical Relativistic Fields, Part II: Canonical Analysis of Field Theories (1999).

${ }^{10}$ D. Cartin, "Generalized symplectic manifolds," dg-ga/9710027.

${ }^{11}$ M. McLean and L. K. Norris, "Covariant field theory on frame bundles of fibered manifolds," J. Math. Phys. 41, $6808-6823(2000)$.

${ }^{12}$ A. Echevarría-Enríquez, M. C. Muñoz-Lecanda, and N. Román-Roy, “Geometry of Lagrangian first-order classical field theories," Fortschr. Phys. 44, 235-280 (1996).

${ }^{13}$ A. Echevarría-Enríquez, M. C. Muñoz-Lecanda, and N. Román-Roy, "Multivector fields and connections: Setting Lagrangian equations in field theories,' J. Math. Phys. 39, 4578-4603 (1998).

${ }^{14}$ A. Echevarría-Enríquez, M. C. Muñoz-Lecanda, and N. Román-Roy, "On the multimomentum bundles and the Legendre maps in field theories,' Rep. Math. Phys. 45, 81-105 (2000).

${ }^{15}$ A. Echevarría-Enríquez, M. C. Muñoz-Lecanda, and N. Román-Roy, "Multivector field formulation of Hamiltonian field theories: equations and symmetries," J. Phys. A 32, 8467-8484 (1999).
} 
${ }^{16}$ A. Echevarría-Enríquez, M. C. Muñoz-Lecanda, and N. Román-Roy, "Geometry of multisymplectic Hamiltonian firstorder field theories," J. Math. Phys. 41, 7402-7444 (2000).

${ }^{17}$ G. Giachetta, L. Mangiarotti, and G. Sardanishvily, "Covariant hamilton equations for field theory," J. Phys. A 32, 6629-6642 (2000).

${ }^{18}$ S. P. Hrabak, "On a multisymplectic formulation of the classical BRST symmetry for first order field theories. Part 1: Algebraic structures," math-ph/9901012.

${ }^{19}$ S. P. Hrabak, "On a multisymplectic formulation of the classical BRST symmetry for first order field theories. Part 2: Geometric structures," math-ph/9901013.

${ }^{20}$ I. Kanatchikov, "Towards a pre-canonical quantization of gravity without the space+time decomposition," preprint 2000.

${ }^{21}$ J. K. Lawson, A frame bundle generalization of multisymplectic geometries, Rep. Math. Phys. 45, 183-205 (2000).

${ }^{22}$ M. de León, E. Merino, J. A. Oubiña, P. Rodrigues, and M. Salgado, "Hamiltonian systems on $k$-cosymplectic manifolds,' J. Math. Phys. 39, 876-893 (1998).

${ }^{23}$ C. Albert, "Le théoreme de réduction de Marsden-Weinstein en géometrie cosymplectique et de contact," J. Geom. Phys. 6, 627-649 (1994)

${ }^{24}$ F. Cantrijn, M. de León, and E. A. Lacomba, "Gradient vector fields on cosymplectic manifolds," J. Phys. A 25 , 175-188 (1992).

${ }^{25}$ D. Chinea, M. de León, and J. C. Marrero, “The constraint algorithm for time-dependent Lagrangians,” J. Math. Phys. 35, 3410-3447 (1994).

${ }^{26}$ A. Morimoto, "Liftings of some types of tensor fields and connections to tangent $p^{r}$-velocities," Nagoya Math. J. 40, 13-31 (1970).

${ }^{27}$ Ch. Günther, "The polysymplectic Hamiltonian formalism in field theory and calculus of variations I: The local case," J. Diff. Geom. 25, 23-53 (1987).

${ }^{28}$ M. de León, I. Méndez, and M. Salgado, “p-almost tangent structures," Rendiconti del Circolo Matematico di Palermo, Serie II, Tomo XXXVII, 1988, pp. 282-294. 